\title{
Software
}

\section{Acrobat 8: la nueva apuesta tecnológica de Adobe para la gestión de documentos en la empresa}

\author{
Por Lluís Codina y Ricard Monistrol
}

\begin{abstract}
Resumen: El formato PDF, desarrollado por Adobe, pero convertido en un estándar abierto y de tipo multiplataforma recibe un nuevo impulso con la versión 8 de Acrobat de la misma empresa. Se revisan sus principales características y se destacan las nuevas funcionalidades de esta nueva aplicación de Adobe que probablemente tendrá un gran impacto en la gestión de documentos electrónicos en las empresas.

Palabras clave: PDF, Documentos electrónicos, Adobe, Acrobat, Revisión de documentos

\section{Title: Acrobat 8: Adobe's new technological wager for business document management}

Abstract: The PDF format, developed by Adobe and since converted to an open, multiplatform standard, gets a new boost from version 8 of Acrobat, produced by the same company. A review is offered of the main characteristics and functionality of this new application that most likely will have a great impact on electronic document management for business.

Keywords: PDF, Electronic documents, Adobe, Acrobat, Document revision

Codina, Lluís; Monistrol, Ricard. "Acrobat 8: la nueva apuesta tecnológica de Adobe para la gestión de documentos en la empresa". En: El profesional de la información, 2007, marzo-abril, v. 16, n. 2, pp. 160-166.

DOI: 10.3145/epi.2007.mar.12

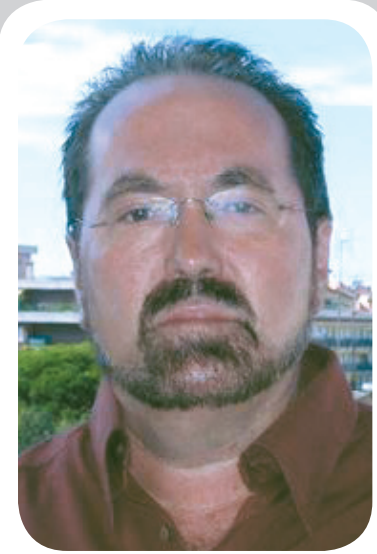

Lluís Codina es doctor en ciencias de la información y profesor titular de ciencias de la documentación de la Univ. Pompeu Fabra (UPF) de Barcelona. Coordina el grupo de investigación en documentación digital (DigiDoc) del Instituto Universitario de Lingüística Aplicada (IULA) de la UPF.

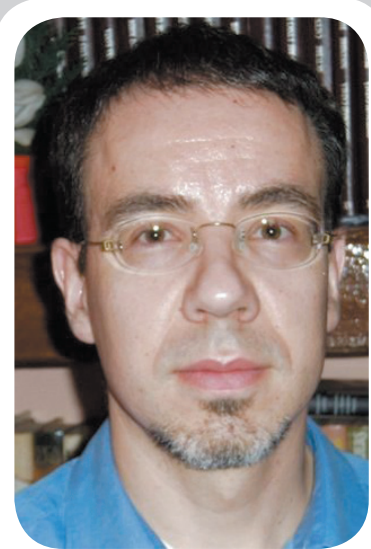

Ricard Monistrol, licenciado en periodismo por la Univ. Autónoma de Barcelona (UAB), diplomado en estudios avanzados en comunicación social e investigador colaborador del grupo DigiDoc, IULA, UPF; es director de Culturcom, consultoría en comunicación y difusión cultural.

\section{EI formato PDF}

COMO ES SABIDO, si una empresa 0 un profesional con gran uso de las comunicaciones electrónicas desconfía sobre el estado en que llegará la documentación a un determinado receptor y quiere estar seguro que se imprimirá o visualizará de forma muy similar con independencia de la plataforma o sistema operativo utilizado por el receptor, puede optar por exportar esa documentación al formato pdf (portable document format).

¿La razón? No solamente que casi el 90\% de los ordenadores presentes en el mundo dispone (entre otros) de Adobe Reader, el lector 


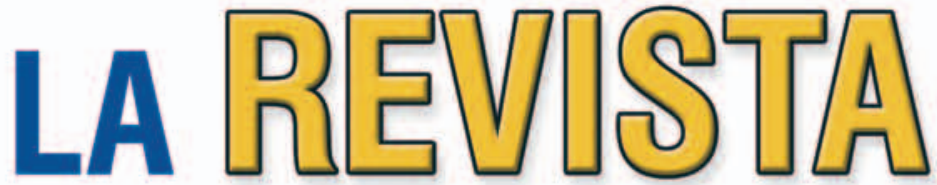 \\ DEL MUNDO DE LAS BIBLIOTECAS}

uiblioteca

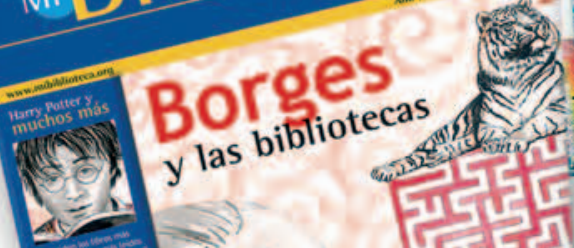
vilblioteca. Miblioteca 4 (1)
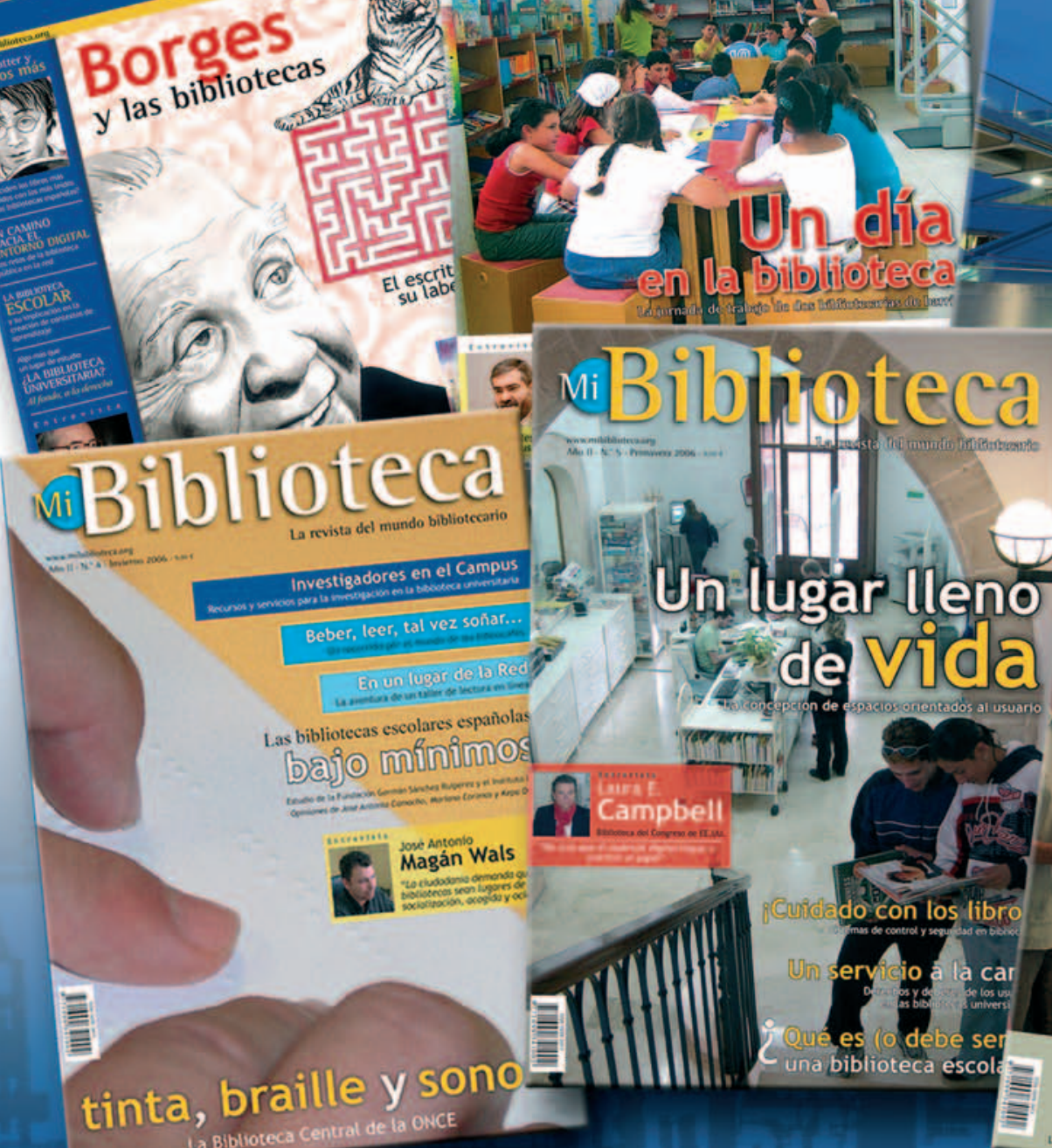
Nibiblioteca Ant

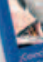


La cuestión es, como señalábamos anteriormente, que pdf ha sido reconocido ya internacionalmente como formato ISO, algo que según sus responsables asegura la supervivencia de los documentos, quizá no para la eternidad, pero sí durante décadas. No es una cuestión trivial: documentos elaborados en otros programas de versiones anteriores actualmente son ilegibles en los formatos actuales, por no hablar de la imposibilidad de abrir documentos creados con aplicaciones distintas, o con la misma aplicación pero de plataformas distintas.

\section{Adobe Acrobat Professional 8}

A inicios de 2007, la empresa Adobe presentó el programa Adobe Acrobat Profesional versión 8, pre- cisamente pocos meses después de haber absorbido ya oficialmente la empresa Macromedia (y después de que tanto la UE como EUA otorgaran sus respectivos permisos).

Había suscitado interés comprobar cómo funcionaban las sinergias de ambas empresas. En diversos productos de Adobe se ha sentido la integración de la tecnología Flash de Macromedia, como por ejemplo Adobe Captivate 2, programa de creación de simulaciones interactivas, demostraciones de software y módulos de formación. Pero no es éste el caso de las aplicaciones "clásicas" de Adobe Acrobat Professional, aunque sí permita acceder desde la misma aplicación a un entorno llamado Adobe Acrobat Connect, una plataforma de videoconferencia virtual (ver recuadro).

\subsection{El organizador de docu-} mentos

Las principales mejoras y novedades de Adobe Acrobat Profesional 8 radican en la indización y en las funciones de búsqueda, que se complementan con el Organizador de documentos.

¿Cuál es la función de este organizador? Accesible desde un desplegable denominado Archivos, o desde el icono que muestra un archivador, el Organizador de documentos permite saber qué documentos pdf (de cualquier origen) se han usado o recibido el mismo día así como en diversas pautas temporales ( 7 días, 14 días, 30 días y un año). Asimismo, posibilita guardar en unos archivos llamados Colecciones aquellos documentos que por razón de afinidad sea útil tenerlos accesibles

\section{Adobe Acrobat Connect}

¿Qué le parecería tener una sala de reuniones virtual que le permitiera tener a la vez 15 participantes interactivos (en la versión Standard) o hasta 2.500 personas (en la versión Professional)? Es la nueva oferta que Adobe, mediante la tecnología Macromedia Breeze ofrece para que cualquier empresa pueda establecer reuniones internacionales desde los distintos puestos de trabajo o sedes de la misma. Aunque no hemos tenido oportunidad de poner a prueba esta opción, se trata de una cuestión nada desdeñable si hacemos caso a la documentación oficial del programa Adobe Acrobat 8. A continuación presentamos una muestra de sus posibilidades (siempre según la documentación oficial del programa):

- Disponer de un ilimitado número de diferentes salas "virtuales" de reuniones (versión profesional).

- Personalizar la URL de cada una de las salas de reunión.

- Pantallas compartidas por diferentes usuarios.

- Chat, bloc de notas e incluso pizarra. Todo ello interactivo y participativo.
- Videoconferencia com imagen (versión normal) y también con voz.

- Voz integrada en protocolo de internet (VoIP).

- Ventanas y presentaciones adaptables a las necesidades del cliente.

- Un mismo usuario puede acceder a diversas salas virtuales.

- Gestión del sistema en manos del cliente, tanto en administración como en informes.

- Posibilita grandes eventos o encuestas.

- Acceso a la biblioteca o base de datos central (datos, documentación, aplicaciones...)

- Posibilidad de crear y añadir diversos elementos (diagramas, hojas de cálculo, textos...) que aumenten el atractivo de presentaciones, seminarios, formación on line, reuniones de trabajo...

En resumen, es realizable cualquier necesidad de comunicar, informar, formar o simplemente trabajar en grupo en un entorno virtual y con todas las herramientas que sean precisas, todo ello a partir de un ancho de banda 1 Mbps. 


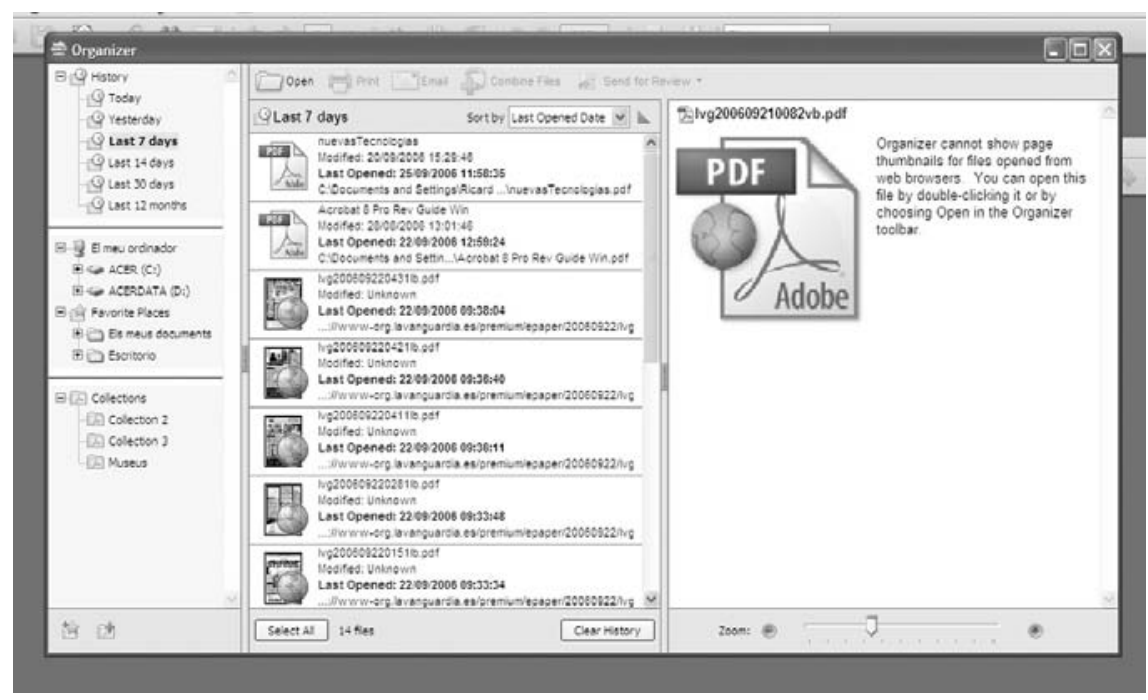

Figura 1: El Organizador de Acrobat 8, donde se aprecia a la izquierda el historial, acceso a los archivos y las colecciones creadas, en el centro los archivos utilizados en los últimos 7 días y a la derecha la visualización del archivo seleccionado mediante zoom.

en la misma carpeta. Simplemente con el mismo ratón, se arrastraran hasta su nueva carpeta en el Organizador. Pero con una salvedad: no variará en nada la ubicación original del archivo físico, que podemos ver mediante el botón derecho del ratón, pero siendo ahora fácilmente accesible desde la ya citada $\mathrm{Co}$ lección etiquetada oportunamente. Además, permite imprimir los documentos seleccionados, gestionar su revisión compartida, combinarlos o enviarlos por e-mail.

¿Qué se podría mejorar en el Organizador? Cualquier usuario o usuarios que gestionen y produzcan un elevado número de documentos, puede gastar demasiado tiempo en buscarlos luego y guardarlos en la colección. ¿Por qué no habilitar una herramienta de búsqueda en el propio Organizador? Su función aumentaría la rapidez en la gestión de archivos, pues buscando por palabras clave los documentos referidos a un tema de revisión, proyecto acabado, informe, etc., se podrían seleccionar los documentos que deseáramos y traspasarlos en conjunto (o individualmente) a una determinada colección. Con todo, debe señalarse que desde el servicio de búsqueda centralizado de la aplicación sí que es posible buscar en las Colecciones. era patrimonio de Windows Microsoft Word, Excel y Power Point.
Asimismo, uno de los problemas que tenía Acrobat 7 era no soportar determinados formatos de documentos a la hora de fusionarlos en un solo pdf, o una cierta facilidad para "colgarse", ya que a veces quedaba bloqueado y era necesario reiniciarlo de nuevo. Con la nueva versión, realizada la prueba con los mismos documentos que provocaban antes el bloqueo, hemos comprobado que la compatibilidad ha mejorado.

Así mismo, en la anterior versión 7 habíamos detectado que introducir un vínculo hipertextual hacia otro archivo o hacia un sitio web aún resultaba complicado por el número de operaciones que debían hacerse, es decir, la usabilidad de esta función era un tanto baja. En la nueva versión esto ha quedado gratamente solventado con la posibilidad de crear a voluntad una o diversas barras de herramientas con todas la funciones que se deseen tener a mano y, de este modo, evitar un buen número de pasos.

De todas formas, probablemente, una de los principales puntos fuertes de Adobe Acrobat 8 es que

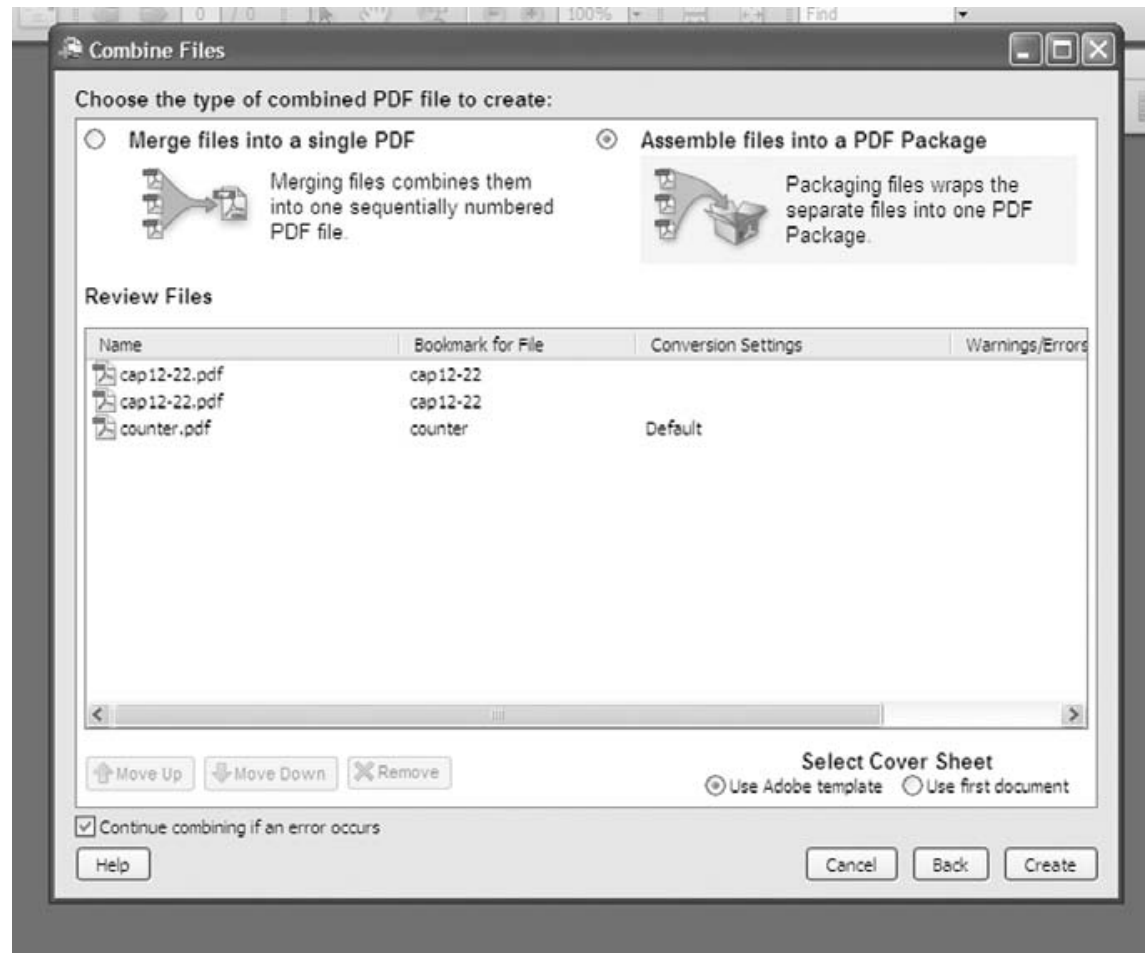

Figura 2: Combinación de archivos, donde se aprecia la mejorada posibilidad de fusión o la innovación en el ensamblaje. 
cualquier documento puede ser revisado y comentado por otras personas, siempre conservando el formato pdf. En la versión anterior (7) esta función no estaba muy resuelta: se conseguía mediante el uso del correo electrónico. Es decir, se enviaba la documentación, se recibían los comentarios nominalizados, y después se integraban al documento.

Ahora, en la versión 8 se ha dado un paso adelante hacia la revisión en tiempo real: ver inmediatamente todos los comentarios una vez han sido realizados. Ahora bien, para esto es necesario que todos los integrantes o miembros del grupo de revisión estén conectados al servidor de red de la empresa o institución. Es una mejora que incrementa la productividad, pues al no depender de un correo electrónico, el documento llega a su fase final en menor tiempo. Además, se aumenta la integración del equipo de trabajo.

Adicionalmente, existe la posibilidad de habilitar el Adobe Reader 8 (recordamos que en este caso es un lector gratuito) para participar en las revisiones compartidas. Es decir, podrá disponer de una completa barra de herramientas de revi- sión, y así ampliar de forma notable el número de personas que pueden participar.

Si hasta ahora hemos estado hablando de la revisión de documentos, la nueva versión es capaz de exportar a Microsoft Word el documento ya finalizado, conservando su diseño, fuentes, formato y tablas, y siendo editable sin ningún tipo de problema.

También cabe destacar que cualquier documento, sea escrito de forma manual o mediante impresora y escaneado, es automáticamente reconocido por la nueva versión de Acrobat, pues está dotado de OCR (optical character recognition). Por ello permite pasar a pdf documentación que nos interese conservar o enviar en formato original y validada con la firma electrónica.

Por último, resulta interesante la característica de activar formularios a partir de un documento que previamente no ha estado diseñado para ello: detecta los posibles campos a rellenar en documentos escaneados, con formato pdf, Word y hoja de cálculo Excel. Una opción útil para la creación y gestión rápida de formularios en cualquier función.

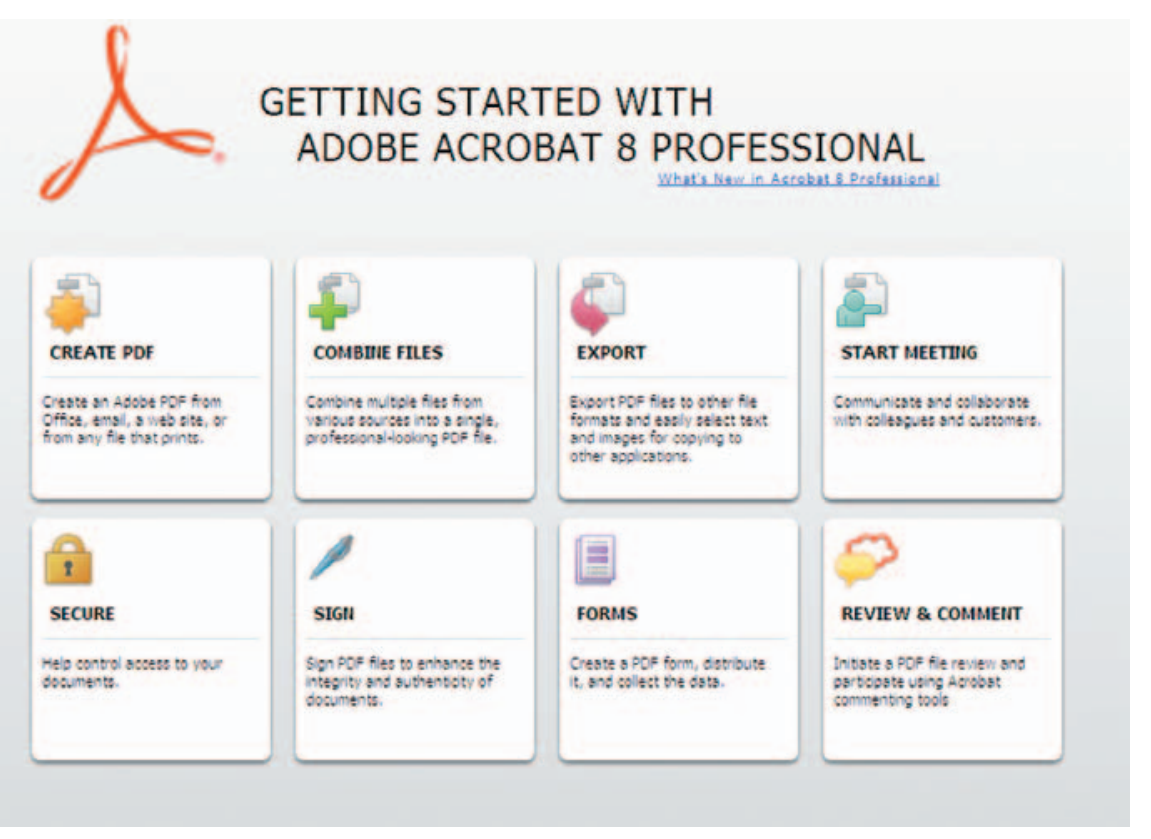

Figura 3: Asistente: función adscrita al inicio del programa, que ofrece acceso rápido y tutorizado a 8 posibles tareas.

\subsection{Innovaciones para la Red y racionalización}

Una de las grandes novedades de Acrobat 8 es, sin duda, el Adobe Acrobat Connect, para celebrar reuniones virtuales en tiempo real, que incorpora varias herramientas que facilitan la comunicación interactiva, como se explica en el recuadro que acompaña este artículo.

Otra novedad es la de poder crear un fichero común donde todos los documentos que lo integran (pasados anteriormente a formato pdf) conserven íntegramente todas sus características. Es decir, se conservarán sus condiciones de seguridad, firmas electrónicas y otros elementos previos a la combinación. Debemos diferenciar esta función de la de fusión de documentos, explicada en el anterior apartado sobre mejoras, con la que los documentos originales pierden sus características al crear uno nuevo que reúne toda la información.

Esta innovación resulta muy útil en aquellos casos en los que al enviar un archivo con algún proyecto, presupuesto o informe sea necesaria la certificación e inviolabilidad de algunos de los documentos con valor jurídico que lo compongan.

En relación con la usabilidad, Adobe destaca como innovación la racionalidad del nuevo diseño de la interfaz, y, sobre todo, la rapidez con la que se pueden realizar las funciones. Se trata de una cuestión clave, pues hemos de entender la "usabilidad" como la capacidad que cualquier aplicación informática facilite al máximo el acceso a una función determinada y a su desempeño de forma sencilla. Es decir, que no haga "pensar" más de lo estrictamente necesario obtener el resultado deseado. ¿Beneficio? Un aumento de la productividad: todo el tiempo que se gaste en aprender a usar una función de un programa se pierde para su verdadero cometido. 


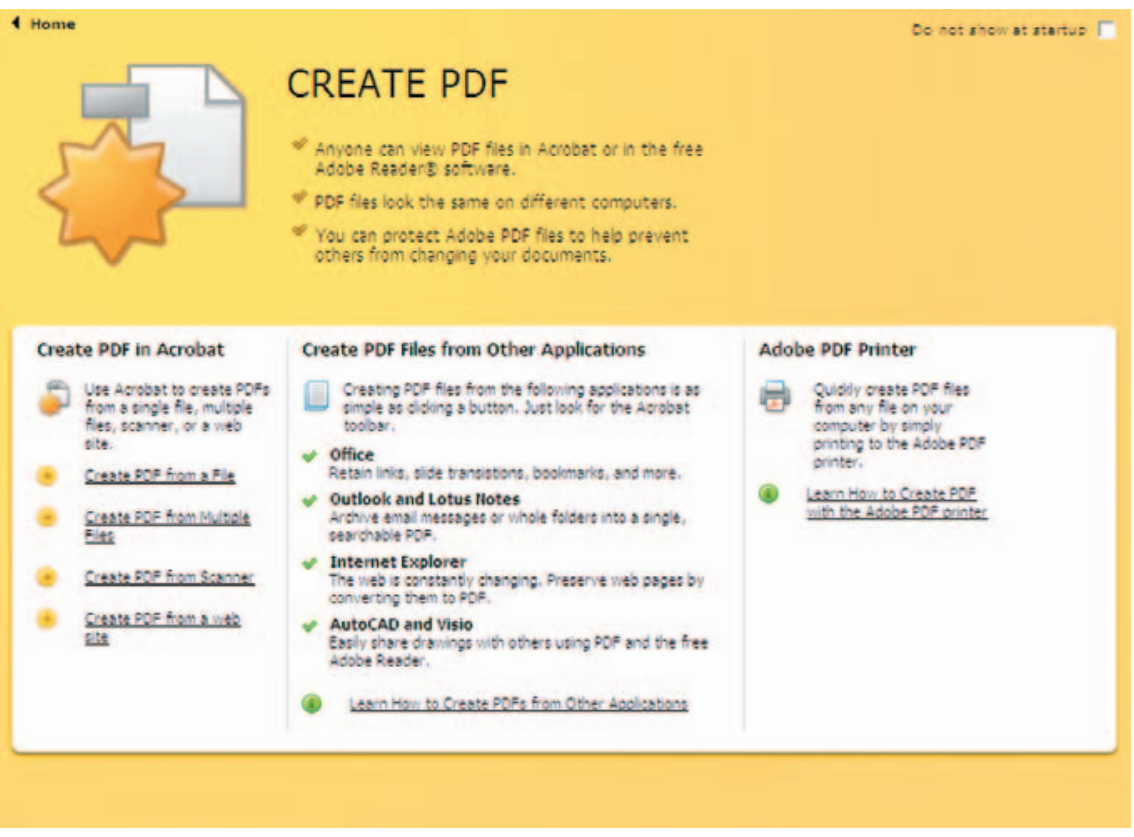

Figura 4: Una de las tareas del Asistente: crear un archivo pdf.

¿Cuáles son los elementos de esta anunciada racionalización? En nuestra opinión el más efectivo es el nuevo asistente de Adobe Acrobat. Se ha trabajado muy bien el aspecto gráfico, y es claro a primera vista. Su disposición de acceso a las funciones se plasma en 8 recuadros, todos ellos formados por un icono descriptivo, por palabras clave y una breve explicación de las funciones. En conjunto se cubre la totalidad de tareas que un usuario normal o avanzado necesita, a saber:

1. Creación de un documento o archivo pdf.

2. Combinación de archivos o documentos.

3. Exportación a otros formatos: permite guardar una aplicación pdf, por ejemplo, en html, jpeg, tiff, doc o xml.

4. Iniciar una reunión virtual mediante la aplicación Adobe Acrobat Connect Professional.

5. Seguridad de los archivos.

6. Firmar documentos digitalmente.

7. Crear formularios.

8. Revisar y comentar archivos.
Tras haber seleccionado alguna función, cada uno de los recuadros remite un menú contextual con explicaciones e instrucciones, donde se describen todas las posibles funciones que puedan ir asociadas a la tarea.

Hasta ahora insertar más o menos elementos de trabajo en las diversas barras de herramientas chocaba con el espacio predeterminado para ello. Ahora hay barras flotan- tes que se pueden colocar en cualquier parte de la pantalla, y con las funciones específicas que se deseen escogidas mediante un menú.

Tal como decíamos anteriormente en el apartado de mejoras, en la versión actual la dificultad de introducir vínculos hipertextuales se ha solventado, pues esa función puede ponerse en la propia barra de herramientas (específicamente la de edición avanzada).

Otra función, que más que mejorada ha sido renovada, es la de búsqueda avanzada a la que también nos hemos referido antes brevemente. Según indica Adobe, a la hora de realizar búsquedas se tienen en cuenta datos que hasta ahora permanecían ocultos: metadatos del documento (en xml); anotaciones realizadas en las revisiones; archivos adjuntos (siempre que estén en pdf); campos que formen un formulario; capas o niveles que formen un documento o archivo; y marcadores (elementos hipertextuales que permiten acceder directamente a una parte del documento previamente seleccionado). Una vez realizada la búsqueda se propone editar o borrar el contenido seleccionado.

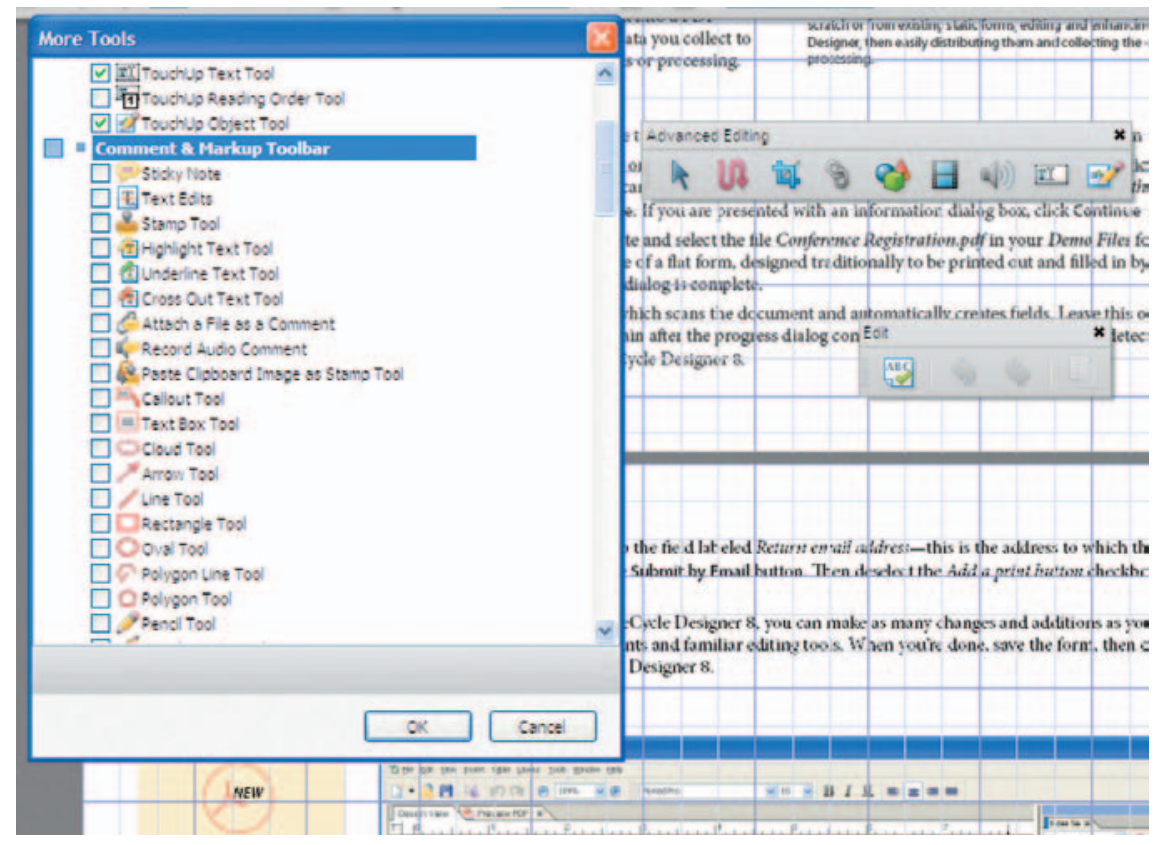

Figura 5: Menú de creación de barras de herramientas; a la derecha aparecen dos de las barras creadas, la de edición y la de edición avanzada. 
Aunque podamos modificar las condiciones de seguridad de un documento presente en internet, es decir, de acceso público, puede resultar relevante para una empresa que ciertos datos no aparezcan, como por ejemplo algún dato sensible que contravenga la Ley Orgánica de Protección de Datos.

Para prevenir contingencias de este tipo, mediante la herramienta de edición avanzada se pueden borrar de forma definitiva texto y/o ilustraciones. Eso no quiere decir que el documento original pierda de forma definitiva los datos, sino que la copia del documento de acceso público no tiene ninguno de ellos. Incluso usando un programa de recuperación de datos, resultará imposible acceder a los contenidos borrados.

$\mathrm{Si}$ antes destacamos que se había mejorado la detección de campos de un documento no diseñado como formulario, Acrobat 8 profesional permite que en un documento pdf estático esos mismos campos se activen de forma automática. De esta forma se obtiene un documento interactivo que puede ser rellenado por otros usuarios, incluso por los que dispongan solamente de Adobe Reader (7 u 8).
Pero no acaban aquí las ventajas: una vez todos los usuarios hayan contestado, Acrobat 8 permite en una sola pantalla tener a la vista cualquiera de los formularios rellenados $\mathrm{y}$, en la parte superior, tenerlos ordenados por nombre $\mathrm{y}$ apellidos. De esta forma se accede a cada uno de los formularios nominalizados, sin cambiar de pantalla. Se puede guardar el archivo en este mismo formato.

\section{Conclusiones}

Como se ha visto, el Adobe Reader 8 realiza funciones nuevas, tales como revisiones compartidas o rellenar formularios (o permitiendo guardar éstos en local para ser rellenados posteriormente).

Resulta muy interesante la opción de Adobe Reader para firmar digitalmente un documento, habilitando además que la firma pueda ser editada con elementos estéticos o simplemente corporativos. No es desdeñable que, además, la Administración haya aceptado que la firma digital de un documento pdf esté asociada al DNI electrónico, lo cual es una gran ventaja para la burocracia oficial, y en el futuro para todo tipo de transacciones. Con su nueva versión Adobe parece haber vuelto a tomar el control de la documentación electrónica en las empresas, pero... ¿es que dejó de tenerlo alguna vez?

Todo parece indicar que la versión 8 de Adobe marcará un cierto “antes y después". Para decirlo de forma simbólica, el salto que representa el número de versión $\mathrm{X}$, ahora, tal vez correspondería más denominarse Adobe v. 10.

\section{Bibliografía}

"Adobe Acrobat". Wikipedia. Consultado: 9-22007.

http://en.wikipedia.org/wiki/Adobe_Acrobat

"Adobe Reader". Wikipedia. Consultado: 9-22007.

http://es.wikipedia.org/wiki/Adobe_Reader

Padova, Ted. Adobe Acrobat 7 PDF bible. Indianapolis: Wiley, 2005.

Steward, Sid. PDF hacks: 100 industrial-strenght tips and tools. Sebastopol: O'Reilly, 2004.

\section{Lluís Codina, Universidad Pompeu Fabra \\ lluis.codina@upd.edu \\ http://www.lluiscodina.com}

Ricard Monistrol, periodista y consultor independiente rmonistrol@gmail.com http://www.ricardmonistrol.cat

\section{Directorio EXIT}

Estimado lector: Por el simple hecho de estar leyendo El profesional de la información demuestra tener especial interés $y$, probablemente, buenos conocimientos sobre algún aspecto de esta variada profesión. Sólo con que haya publicado algún artículo o pueda impartir un par de horas de clase sobre su trabajo usted merece ser aceptado en el Directorio de Expertos en el Tratamiento de la Información.

Rellene hoy mismo su ficha y sea miembro de una comunidad internacional de 800 expertos capaces de participar en cursos, mesas redondas, proyectos de investigación, etc.

http://directorioexit.info 